\title{
Esophageal Hemorrhage, CTCAE
}

National Cancer Institute

\section{Source}

National Cancer Institute. Esophageal Hemorrhage, CTCAE. NCI Thesaurus. Code C143453.

A disorder characterized by bleeding from the esophagus. 